\title{
Individual Differences in the Use of Simplification Strategies in a Complex Decision-Making Task
}

\author{
James Onken, Reid Hastie, and William Revelle \\ Northwestern University
}

\begin{abstract}
Decision time results were used to assess the strategies that 90 college undergraduates used in a complex decision-making task. Trend analyses revealed that the functions relating choice time to the number of choice alternatives in a set and the number of attributes comprising those alternatives contained linear (increasing) components. In addition, for a portion of the subjects, there was a quadratic effect of the number of attributes available to the decision maker on choice time, suggesting that these subjects adopted simplification strategies at high levels of task complexity. Reliable individual differences in these trend components were observed, consistent with individual differences in motivation and/or processing capacities. These individual differences were included in an information-processing model of decision behavior that captured the choice time data observed in this study. Subjects' ratings of apartments were used as a basis to assess the extent to which the use of simplification strategies resulted in preference reversals. Contrary to expectation, subjects whose choice times contained quadratic components demonstrated fewer preference reversals at high levels of information load.
\end{abstract}

The effect of task complexity on strategy selection has been examined in several studies of contingent decision behavior (Payne, 1982). This research has demonstrated that linear, compensatory models of decision making, such as a multiattribute utility (MAU) model, do not accurately describe choice processes when decision makers must process large amounts of information. The failure of linear models as process descriptors is often attributed to the demands placed on the human information-processing system by the use of strategies derived from these models (Shugan, 1980a, 1980b; Simon, 1955, 1979). In order to reduce the cognitive demands of choice tasks, individuals often utilize simpler (nonlinear) decision strategies (Einhorn, 1971; Payne, 1976; Wright, 1975).

This study formed the basis of the senior author's masters thesis completed at Northwestern University and was supported, in part, by Grant MH29209 from the National Institute of Mental Health.

The principal author would like to extend special thanks to Peter Frey for his comments relevant to this study. He also gratefully acknowledges the assistance of Joe DeMuyt for help in running subjects and the aid of several graduate students at Northwestern who served as pilot subjects.

Requests for reprints should be sent to James Onken, who is now at AT \& T Bell Laboratories, Warrenville and Naperville Roads, Naperville, Illinois 60566.
Cost-benefit models of the strategy selection process have been developed in which the costs of processing information (in terms of mental effort and time) are weighed against the utility of making an accurate choice (Christensen-Szalanski, 1978, 1980; Hogarth, 1975; Pollay, 1970; Russo \& Dosher, 1983). From a cost-benefit perspective, decision makers attempt to minimize the joint costs of processing information and of making inaccurate decisions. When the cognitive demands of a task are great and the utility of making an accurate choice does not justify the use of a complex decision-making strategy, the decision maker uses a simpler decision strategy that allows him or her to decrease the cognitive demands of the task without costly errors in judgment.

Hogarth (1975) proposed a model of decision time incorporating cost-benefit considerations. The model accounts for findings which suggest that the time it takes a decision maker to reach a decision is a curvilinear function of task complexity (Hendrick, Mills, \& Kiesler, 1968; Kiesler, 1966; Pollay, 1970). In this model, task complexity is a function of the number of attributes per alternative and the difficulty of the choice task, operationally defined in terms of the number of common characteristics of the alternatives 
under consideration. The greater the number of common characteristics, the easier the decision task.

According to this model, increases in task complexity affect the decision-making process through increases in the amount of cognitive strain induced by the task. Cognitive strain is postulated to be a convex function of task complexity; as either the number of dimensions per alternative or the difficulty of the decision task increases, cognitive strain increases at an accelerating rate. The slope of the function relating cognitive strain to either factor of task complexity is assumed to be equal to unity when the processing limitations of the decision maker have been met. Beyond these limitations, a unit increase in task complexity produces an even greater increase in cognitive strain.

The total cost of time invested in the decision-making process is a function of both cognitive strain and the amount of time already spent on the decision task. The amount of time spent on the task is also related to the probability of making an error; fewer errors are made as the amount of time spent deciding increases. The optimal strategy for a decision maker is to invest that amount of time in the decision process that minimizes a joint function of the costs of time invested in the task and the cost of errors.

Under these assumptions, it can be shown that the optimal decision time will increase with increases in task complexity until the processing capacities of the decision maker have been reached (Hogarth, 1975). Increases in task complexity beyond this point will produce relatively large increases in cognitive strain, and the marginal costs of processing time will be great. As a result, increasingly rapid decisions as well as more errors are predicted as task complexity increases beyond the processing capacities of the decision maker.

Describing the effect of increases in task complexity on the decision-making process is not made without at least implicit reference to characteristics of the decision maker that might moderate the impact of these manipulations. From the perspective of a costbenefit model, these individual differences are likely to appear either as differences in the cost of making a decision or as differences in the utility of making accurate choices. For example, the costs of processing information might be a function of working memory capacity, the speed with which information is accessed in long-term memory, or the speed with which elementary processing operations are performed. Processing costs will be greatest for those individuals who possess relatively limited processing abilities, and these individuals may show a greater tendency than others to utilize simplification strategies as the complexity of a decision task increases. Similarly, decision makers who perceive little utility in making accurate choices are also more likely to use simple decision strategies.

Individual differences that affect strategy selection will also be reflected in the response of decision time to increases in task complexity. Hogarth's cost-benefit model of decision time suggests that individuals having limited processing capacities or lower motivation will begin to trade off accuracy for speed at a lower level of task complexity than will individuals with greater processing abilities or motivation.

The role of individual difference variables such as processing abilities or motivation in decision-making processes has been a neglected area of research despite indications that such issues deserve attention (Einhorn, 1970; Svenson, 1979). The research reported here examined the extent to which reliable individual differences exist in the response of decision speed and accuracy to increases in task complexity.

An attempt was also made to identify sources of task complexity. It is not clear to what extent task complexity is a function of the number of alternatives in a choice set, the number of attributes comprising those alternatives, or some multiplicative relationship between these variables. The contribution of each of these factors to total task complexity determines the effect of each on decision time. There is conflicting information, both theoretical and empirical, about two points: (a) that decision time, typically defined as the time elapsed from the presentation of the choice alternatives to the decision-maker's choice, is nonmonotonically related to the number of choice alternatives (Jacoby, Speller, \& Berning, 1975; Kiesler, 1966) and (b) that this relationship is monotonically increasing 
(Hendricks et al., 1968; Hogarth, 1975). Payne and Braunstein (1978) made a distinction between the amount of time used to gather information and the amount of time spent deciding after all information used to make a decision had been searched. The results of their study suggested that both search and decision time decreased as the number of choice alternatives increased. There is also evidence to suggest that decision time is a nonmonotonic function of the number of dimensions along which the alternatives vary (Hogarth, 1975; Jacoby et al., 1975) and that this relationship is linearly decreasing (Hendricks et al., 1968).

The effect of the number of alternatives and attributes on decision accuracy is also not clear. Accuracy has been shown to increase as the number of attributes increases (Jacoby, Speller, \& Kohn, 1974; Jacoby, et al., 1975; Wright, 1975) and to decrease with more alternatives (Jacoby et al., 1974, 1975; Wright, 1975). In previous studies, an accurate choice was defined as the selection of an alternative that was most similar to the subject's ideal alternative. However, there is no way of objectively determining what the correct choices are in preferential choice tasks, and it is difficult to identify exactly when an "error" has occurred. The accuracy results described above may not be obtained when accuracy is given a different operational definition. In the present study, all of the choice alteratives used to construct the choice sets were individually rated for their attractiveness by each subject before any choices were made. Accuracy was measured by the extent to which a subject chose, from each choice set, that alternative to which he or she had given the highest rating. Inaccuracy reflected a departure of the rating judgment from the outcome of the choice process, otherwise known as a preference reversal (Grether \& Plott, 1979), and is a measure of intraindividual inconsistency.

\section{Method}

\section{Subjects}

The subjects for this experiment were 99 Northwestern University undergraduates who were completing research requirements for an introductory psychology course. The data from 9 of these subjects were not used because of errors in data collection. The analyses were based on the remaining 90 subjects. For analyses involving questionnaire data, an additional subject was deleted for failing to appropriately complete the questionnaires.

\section{Design}

Choice sets containing either $2,4,8$, or 12 alternatives were constructed from stimuli comprising either $2,4,8$, or 12 attributes. The factorial combination of set size and stimulus complexity resulted in 16 levels of information load. Each subject was presented with 32 choice sets-two choice sets from each level of information load. Trials were organized into two blocks. Each of the 16 levels of information load was presented once in each trial block. Within a block of trials, each level of the independent variables occurred once in each set of four trials.

\section{Materials}

The choice alternatives in this experiment were 60 hypothetical apartments similar to those used by Payne (1976). The values for most of the attributes were expressed as locations along a 3-point scale such as smallmedium-large (size), 10-12-30 $\mathrm{min}$ (distance), poorfair-good (landlord, cleanliness, brightness, and kitchen space), low-moderate-high (noise level and crime), noneel only-el and train (transportation; el is elevated train), and below average-average-above average (closet space). Exceptions were rent, which was expressed as one of five levels ranging from $\$ 260$ to $\$ 350$, and parking, which was either off-street or garage. In addition to these 60 alternatives, 10 additional apartments were generated to be used for practice trials.

The apartments for each choice set were selected from 15 apartments designed at each level of stimulus complexity. Choice sets were presented in booklet form with each of the 32 choice sets on a separate page of standard, lined, 28-cm $\times 38-\mathrm{cm}$ computer printout paper. A choice set similar to those used in this experiment is shown in Figure 1.

In a postexperimental questionnaire, ${ }^{\prime}$ subjects were asked to rank the attributes in order of importance from 1 to 12. They were also asked to outline, in systematic fashion, the decision strategy they most often used to make their decisions. Finally, subjects were presented with brief descriptions of common decision strategies and asked to rank these strategies with respect to the frequency with which each was used. These strategies ranged in difficulty from relatively complex, compensatory strategies, such as a MAU model and an additive difference model (ADM), to simpler strategies such as a lexicographic strategy, elimination by aspects (EBA; Tversky, 1972), a

\footnotetext{
'Pre- and postexperimental questionnaires were constructed to assess demographic variables, patterns of information usage, and strategy choices. The preexperimental questionnaire contained demographic questions that could be related to the subject's use of the various attributes. Although the information derived from this questionnaire is potentially interesting, these data were not analyzed in this experiment.
} 


\begin{tabular}{l|llll|} 
APT. & RENT & SIZE & DISTANCE & LANDLORD \\
\cline { 2 - 5 } & & & & \\
1 & $\$ 325$ & MEDIUM & $10 \mathrm{MIN}$. & $600 D$ \\
2 & $\$ 300$ & SMALL & $20 \mathrm{MNN}$. & 6000 \\
3 & $\$ 350$ & LARGE & $10 \mathrm{MIN}$. & POOR \\
4 & $\$ 260$ & SUALL & $20 \mathrm{MIN}$. & FAIR \\
5 & $\$ 350$ & LARGE & $30 \mathrm{MIN}$. & GOOD \\
6 & $\$ 280$ & MEDUUM & $30 \mathrm{MIN}$. & FAIR \\
7 & $\$ 280$ & SMALL & $10 \mathrm{MIN}$. & POOR \\
8 & $\$ 300$ & LARGE & $30 \mathrm{MIN}$. & FAIR \\
\hline
\end{tabular}

Figure 1. Example of eight-alternative choice set used in this study.

satisficing approach (SAT; Simon, 1955), and a "majority of confirming dimensions" strategy (MCD; Russo \& Dosher, 1983).

All subjects were also given the Eysenck Personality Inventory (EPI; Eysenck \& Eysenck, 1964). However, because the subjects' scores on this test were not of central concern to this study, no analyses were performed on these data.

\section{Apparatus}

Apple II microcomputers were used to present task instructions to subjects as well as to present the 70 apartments, which were to be rated on a 100 -point scale of attractiveness in the rating task. These ratings and rating times were recorded. For the choice portion of the experiment, Apples were used to record the apartment selected from each set and the decision time required to arrive at that choice.

\section{Procedure}

Subjects were run in groups of from 1 to $S$ individuals. The use of microcomputers to record responses allowed subjects to work at their own pace. The amount of time taken to finish the experiment varied, ranging from approximately $\mathrm{l} \mathrm{hr}$ to $\mathrm{I}^{3 / 4} \mathrm{hr}$.

Subjects were first asked to fill out the preexperimental questionnaire and the EPI. Subjects were told by the experimenter that the experiment would comprise two parts, a portion in which they were asked to rate the 70 apartments on a 1 to 100 scale, and a second portion in which they would be presented with sets of apartments and asked to choose that apartment that they liked best from eact set. Subjects were not made aware that their ratings or choices were being timed, and no instructions were given regarding the pace at which they should work.

Control of the experiment was then turned over to the microcomputers. The instructions for the rating task included descriptions and definitions of each attribute along which the apartments varied and the different values these attributes could assume. When a subject had completed the instructions, he or she was immediately presented with the rating task. Ten practice trials were used to familiarize subjects with the use of the response scale before presenting the 60 choice stimuli. Ratings as well as response times were recorded for this portion of the experiment.

Following the rating task, further instructions were given regarding the choice portion of the experiment. All subjects were shown a sample choice set and were instructed to wait after entering each choice for a signal from the computer before turning the page and continuing with the next choice set. This signal activated the response timer and allowed for more accurate records of choice times. ${ }^{2}$ Immediately upon completion of the 32 choice sets, subjects were asked to complete the postexperimental questionnaire.

\section{Results}

Trend analyses were used to examine the response of decision time to changes in the independent variables. The two choice times per cell for each subject were averaged, the data from all 90 subjects pooled, and these data submitted to a 4 (alternatives) $\times 4$ (attributes) ANOVA for trend with repeated measures. This analysis revealed significant linear, $F(1,89)=277.17, p<.01$; quadratic, $F(1$, $89)=28.84, p<.01 ;$ and cubic, $F(1$, $89)=4.57, p<.05$, effects of set size on decision time. The relationship between decision time and the number of attributes comprising each alternative contained significant linear, $F(1,89)=299.20, p<.01$; and quadratic, $F(1,89)=73.37, p<.01$, components. For both linear trends, choice time increased with increases in the independent variable. Both quadratic components resulted from negatively accelerating increases in choice time with increases in the independent variables. These trends are consistent with the predictions of cost-benefit models, suggesting that a decreasing proportion of the additional information was processed as information load increased.

In addition to these simple trends, the number of alternatives moderated the effect of attributes on decision time, resulting in Linear $\times$ Linear, $F(1,89)=35.46, p<.01$; Linear $\times$ Quadratic, $F(1,89)=110.53, p<$ .01 ; and Linear $\times$ Cubic, $F(1,89)=4.01$,

\footnotetext{
${ }^{2}$ Informal discussions with pilot subjects and the subjects used in the experiment suggested that subjects did not realize that apartments used in the rating and choice tasks were identical stimuli.
} 
$p<.05$, interactions. The first of these interactions revealed that the slope of the linear relationship between choice time and the number of attributes increased as the number of choice alternatives in a set increased. The quadratic effect of attributes on decision time also increased with set size, producing a more pronounced inverted U-shaped relationship with larger choice sets. The quadratic effect of alternatives on decision time was also a cubic function of the number of attributes, resulting in a Quadratic $\times$ Cubic interaction, $F(1,89)=16.89, p<.01$. In Figure 2 , mean response time is plotted as a function of the number of attributes for choice sets of varying sizes.

To examine individual differences in the response of decision time to changes in information load, separate trend analyses were performed on each subject's data. A summary in Table 1 shows, for each trend component, the number of subjects in whose data the trend was statistically significant.
The results of analyses performed at the subject level are similar to those obtained from the aggregate data. However, the quadratic effect of set size on decision time is not as prominent in the individual data. From the 90 subjects in this experiment, the data of only 6 showed a significant quadratic effect of alternatives on decision time. This number is close to that expected on the basis of chance alone. The results in this table demonstrate that, for many subjects, the relationship of choice time to the number of attributes comprising the choice alternatives had a nonlinear component that could indicate the use of simplifying strategies at high levels of stimulus complexity.

Decision times collected in each of the two trial blocks were used to obtain split-half estimates of the reliability of individual differences in the size of each trend component. Reliable individual differences were found in several of the trend components: the mean component $\left(r_{x x}=.88\right)$, which is a measure

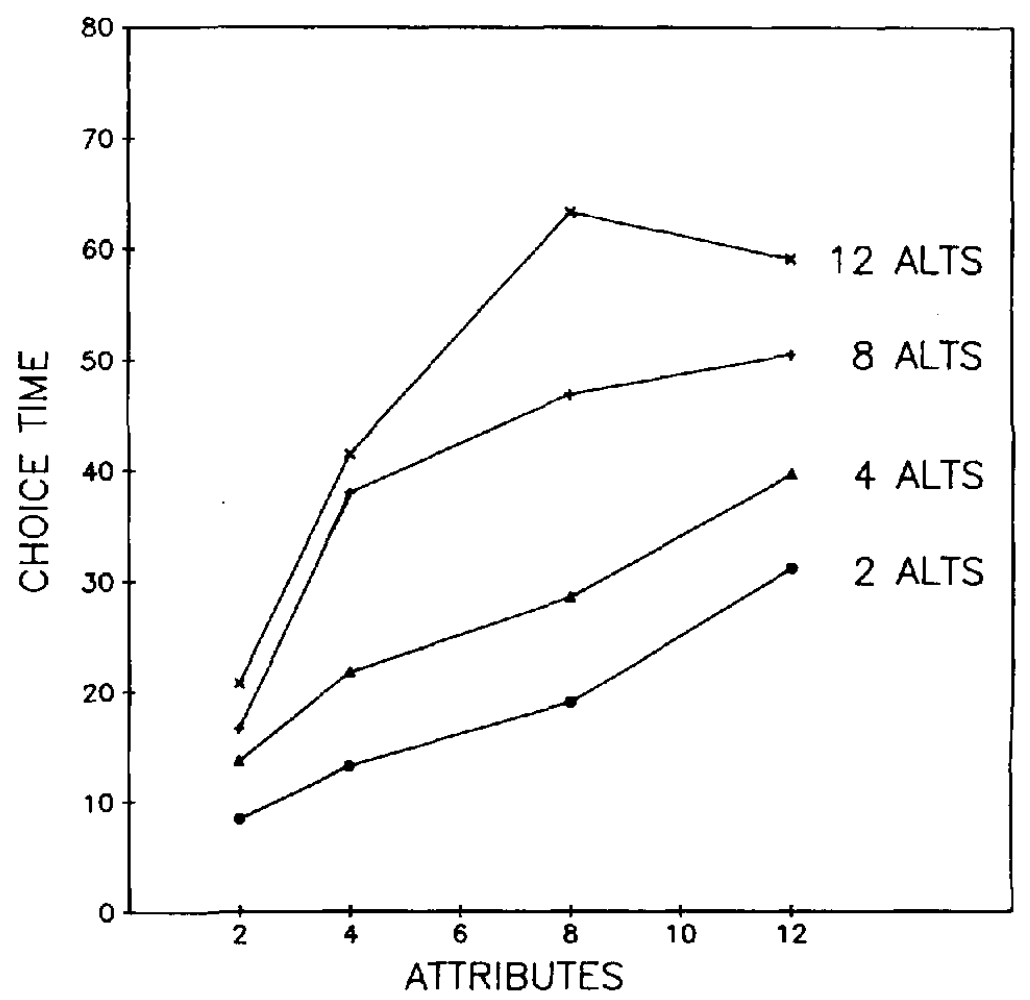

Figure 2. Mean choice time (in seconds) as a function of stimulus complexity (ATTRIBUTES) and set size (ALTS). 
Table 1

Frequency of Trend Components Reaching

Statistical Significance $(p<.05)$

in Subject Data $(N=90)$

\begin{tabular}{cc}
\hline Component & Frequency \\
\hline Alternative & \\
Linear & 80 \\
Quadratic & 6 \\
Cubic & 5 \\
Attribute & \\
Linear & 81 \\
Quadratic & 17 \\
Cubic & 1 \\
Interaction & 18 \\
L $\times \mathrm{L}$ & 16 \\
$\mathrm{~L} \times \mathrm{Q}$ & 1 \\
$\mathrm{~L} \times \mathrm{C}$ & 1 \\
$\mathrm{Q} \times \mathrm{L}$ & 5 \\
$\mathrm{Q} \times \mathrm{Q}$ & 4 \\
$\mathrm{Q} \times \mathrm{C}$ & 3 \\
$\mathrm{C} \times \mathrm{L}$ & 3 \\
$\mathrm{C} \times \mathrm{Q}$ & 3 \\
$\mathrm{C} \times \mathrm{C}$ & \\
\hline
\end{tabular}

Note. $\mathrm{L}=$ linear; $\mathrm{Q}=$ quadratic; $\mathrm{C}=$ cubic.

of average choice time over all levels of information load; the linear $\left(r_{x x}=.81\right)$, quadratic $\left(r_{x x}=.46\right)$, and cubic $\left(r_{x x}=.45\right)$ effects of attributes on decision time; and the linear $\left(r_{x x}=.67\right)$ and cubic $\left(r_{x x}=.35\right)$ effects of set size. There were also reliable individual differences in the Linear $\times$ Linear component $\left(r_{x x}=.36\right)$. These reliabilities suggest that the response of a subject's decision-making strategy to increases in task complexity, reflected in decision time, was consistent across time.

The results presented in Table 1 suggested that the effect of the independent variables on decision time could be described largely in terms of five trend components: linear trends associated with both the number of alternatives and the number of attributes in a choice set, a quadratic effect of attributes, a Linear $\times$ Linear interaction, and a Linear $\times$ Quadratic interaction. However, it can also be seen in the table that all of these trends were not found in the data of each subject. Examination of the individual trend analyses suggested that, on the basis of the five most important trends, subjects could be grouped in accordance with four alternative models of decision time:

1. $\mathrm{L}$ (alt) $+\mathrm{L}(\mathrm{att})$

2. $\mathrm{L}$ (alt) $+\mathrm{L}(\mathrm{att})+\mathrm{L} \times \mathrm{L}$
(46 subjects) (14 subjects)
3. $\mathrm{L}(\mathrm{alt})+\mathrm{L}$ (att) $+\mathrm{Q}$ (att)

4. $\mathrm{L}($ alt $)+\mathrm{L}(\mathrm{att})+\mathrm{Q}(\mathrm{att})+$ $\mathrm{L} \times \mathrm{Q}$

(7 subjects)

(23 subjects).

A subject fit by Model 3, for example, would have a significant proportion of variance in choice time accounted for by linear (L) effects associated with increases in both the number of alternatives (alt) and attributes (att), as well as a quadratic (Q) component associated with increases in the number of attributes.

In a few cases, it was unclear into which group a subject should be placed. For instance, the data of the subject might have contained significant linear effects of both independent variables and a quadratic effect of attributes, as well as a Linear $\times$ Linear interaction. A subject such as this could be grouped with others in Group 2, whose data contained the linear effects and the Linear $\times$ Linear interaction, or with subjects in Group 3, whose data contained the linear effects and a quadratic effect of attributes. In a case such as this, the relative size of the $F$ values corresponding to the quadratic effect and the Linear $x$ Linear interaction would be used to determine whether an individual was fit better by Model 2 or Model 3.

In order to assess the fit of each of the above models to the individual data, omega squares were calculated for each subject. This statistic can be used to indicate the total proportion of variance in a subject's data that is accounted for by the model's components and in this respect is similar to a squared correlation coefficient. The sum of squared omegas across these components can be used as an index of model fit. The mean goodness of fit of each of the above models was $.428, .712, .414$, and .532 , respectively. Mean choice time as a function of task complexity for each set size is presented for subjects fit by these four models in Figure 3.

After grouping subjects in the above manner, we attempted to link group membership with other variables such as patterns of information usage and strategy selection. Of most interest were variables that would distinguish individuals whose choice times had quadratic components (Groups 3 and 4) from those subjects in Groups 1 and 2 whose response times were only linearly related to 
changes in information load. The existence of a quadratic component may be indicative of greater inclination to use simplifying strategies at high levels of information load.

In order to examine possible betweengroup differences in strategy selection, mostpreferred strategies were tabled according to group membership as in Table 2. These were the strategies subjects reported in the postexperimental questionnaire as having been used most often. Due to small cell frequencies, Groups 1 and 2 and Groups 3 and 4 were combined to form one linear and one qua- dratic group, respectively, in these and subsequent analyses. In addition, those subjects who preferred MAU and SAT strategies were eliminated from the analysis because of the small expected frequency of usage of these strategies. The final dataset was submitted to a chi-square, resulting in a nonsignificant result of $3.09, d f=2, N=85, p>.05$, suggesting no differences between these groups in strategy preferences. A similar analysis was performed on second-most preferred strategies. This too resulted in a nonsignificant chi-square, $\chi^{2}(2, N=82)=5.68, p>.05$.
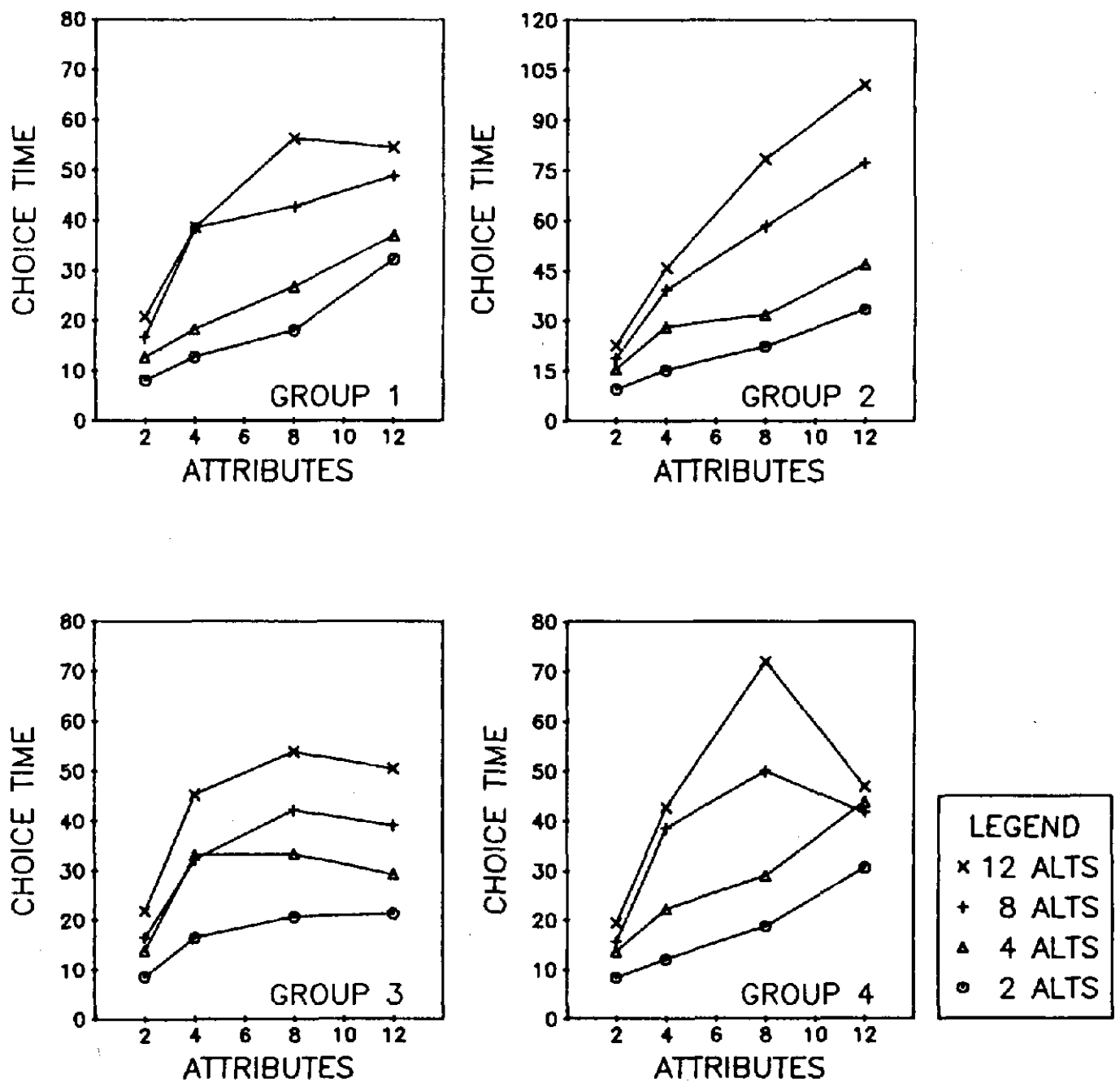

Figure 3. Mean choice time (in seconds) as a function of stimulus complexity (ATTRIBUTES) and set size (ALTS) for subjects grouped on the basis of component trends relating the independent variables to choice time. 
Table 2

Frequency of Preferred Strategies as a Function of Group

\begin{tabular}{lrrrrrr}
\hline & \multicolumn{5}{c}{ Strategy } & \\
\cline { 2 - 5 } \multicolumn{1}{c}{ Group } & MAU & EBA & SAT & MCD & ADM & Total \\
\hline L + L & 3 & 31 & 1 & 5 & 5 & 45 \\
L + L + L $\times$ L & 0 & 9 & 0 & 2 & 3 & 14 \\
L + L + Q & 0 & 6 & 0 & 1 & 0 & 7 \\
L + L + Q + & & & & & & \\
L $\times$ Q & 0 & 20 & 0 & 2 & 1 & 23 \\
\hline
\end{tabular}

Note. $\mathrm{L}=$ linear; $\mathrm{Q}=$ quadratic. $\mathrm{MAU}=$ multiattribute utility, EBA = elimination by aspects, $\mathrm{SAT}=$ satisificing, $\mathrm{MCD}=$ majority of confirming dimensions strategy, ADM $=$ additive difference model.

Greater use of simplification strategies was also expected to result in more decision "errors" or preference reversals, defined as instances in which subjects did not choose that apartment from a choice set to which they had earlier given the highest rating. The accuracy of subjects in Groups 1 and 2 was compared with the accuracy of subjects in Groups 3 and 4. Each decision a subject made was scored by obtaining the difference between the highest rating given to an apartment in the set and the rating of the apartment that the subject actually chose. This difference was then divided by the standard deviation of the ratings given to all apartments in the set to yield a standardized "distance score."

Each subject's two distance scores from each cell were averaged and the resulting data submitted to a 2 (group) $\times 4$ (alternatives) $\times$ 4 (attributes) unweighted means analysis for repeated measures. Cell means are presented in Table 3. There were main effects of the number of alternatives, $F(3,261)=13.69$, $p<.01$; and the number of attributes, $F(3$, $261)=4.60, p<.01$; as well as Alternatives $X$ Attributes, $F(9,783)=2.18, p<.05$; and Group $\times$ Attributes, $F(3,261)=3.32, p<$ .05 interactions. Contrary to what was expected on the basis of the decision time results, Groups 3 and 4 were more accurate than were Groups 1 and 2 when apartments were described by 12 attributes.

\section{Discussion}

The results obtained from both the pooled data as well as from analyses at the subject level suggested a tendency for some subjects to simplify tasks at high levels of information load. This tendency was manifested in quadratic effects of the independent variables on decision time. Although curvilinear relationships alone may not be sufficient to identify the use of simplifying strategies, for several subjects the relationship between choice time and the number of attributes comprising the alternatives was nonmonotonic. This pattern is consistent with cost-benefit approaches to strategy selection (Christensen-Szalanski, 1978), information search (Weitzman, 1979), and decision time (Hogarth, 1975).

There was a quadratic effect of set size on decision time for only a few subjects. Apparently, the number of alternatives in a choice set had less effect than did the number of attributes on the use of simplifying strategies. This result is consistent with Hogarth's model of decision time in which task complexity is a function of the number of attributes comprising each alternative, and decision time is monotonically related to the number of alternatives in a choice set.

Split-half reliability estimates suggested that there were reliable individual differences in the size of several of these trend components. The nature of these individual differences is

Table 3

Preference Reversals as a Function of Attributes and Alternatives for Linear and Quadratic Groups

\begin{tabular}{cccccc}
\hline & \multicolumn{5}{c}{ Attributes } \\
\cline { 2 - 6 } Set size & 2 & 4 & 8 & 12 & $M$ \\
\hline \multicolumn{5}{c}{ Linear group } \\
2 & 0.57 & 0.81 & 0.46 & 0.83 & 0.67 \\
4 & 0.44 & 0.72 & 0.65 & 0.80 & 0.65 \\
8 & 0.66 & 0.96 & 0.99 & 0.90 & 0.88 \\
12 & 0.64 & 1.02 & 0.94 & 1.13 & 0.93 \\
$M$ & 0.58 & 0.88 & 0.76 & 0.92 & 0.78 \\
\hline \multicolumn{5}{c}{ Quadratic group } \\
2 & 0.76 & 0.64 & 0.76 & 0.66 & 0.71 \\
4 & 0.31 & 0.75 & 0.87 & 0.65 & 0.65 \\
8 & 0.84 & 0.99 & 1.07 & 0.62 & 0.88 \\
12 & 0.93 & 1.12 & 1.04 & 0.86 & 0.99 \\
$M$ & 0.71 & 0.88 & 0.94 & 0.70 & 0.81 \\
\hline
\end{tabular}

Note. Entries are mean standard scores obtained by dividing the difference between the highest rating in a set and that of the chosen apartment by the standard deviation of ratings within the set. 
not clear and may be particular to each component. They may be manifestations of individual differences in reading time, the speed with which elementary processing operations are performed, or they may reflect more global constructs such as motivation or processing capacity.

For example, individual differences in the linear component (slope) of the function relating set size to choice time may represent individual differences in the speed with which comparisons are made between alternatives, the rate at which alternatives are rejected from consideration, and so on. The existence of individual differences in the quadratic effect of attributes on decision time suggests that individuals differed in their tendency to simplify decision tasks as task complexity increased. From the perspective of a costbenefit model of strategy selection, these differences might be manifestations of underlying individual differences in the utility of making a correct decision (motivation) or of differences in information processing costs (capacity). Research that is aimed at construct validation is needed before any firm conclusions can be drawn regarding the nature of these individual differences.

To examine some possible differences between individuals in the response of decision time to increases in information load, subjects were grouped in accordance with four alternative models that would describe the effects of information load on decision time. No correlates of group membership could be found in preferred processing strategies, suggesting that these decision time patterns were not related to the use of specific choice strategies. The decision to simplify a complex decision task may have resulted in the modification of a single preexisting strategy through changes in the amount of information sampled, severity of cutoffs, rejection criteria or rejection rates, and so on, rather than in the selection of an alternative processing strategy. This is in contrast to the formulation of Christensen-Szalanski (1978), in which increased decision costs result in the adoption of qualitatively different choice strategies. Increasing decision costs in this experiment may have produced changes only in the parameters of a consistent decision-making process.
The pattern of results obtained when preference reversals were analyzed as a function of group membership suggests that decisions were made more accurately at high levels of complexity by subjects whose choice times were curvilinearly related to complexity. It's possible that this result reflects between-group differences in the way information was processed during the rating task, which could affect the use of the rating scale. On the other hand, the decision task may have been easier for subjects in Groups 3 and 4 when the apartments were described by all 12 attributes. Although this is contrary to intuitive notions of "complexity," such an effect could occur if some of the attributes included at only the greatest level of complexity were particularly important to these subjects. The salience of these dimensions may have increased the discriminability of the choice alternatives, resulting in decisions that were both faster and more accurate.

This finding suggests that a replication of the decision time results found in this study is necessary. It appears that presenting choice stimuli in such a way as to control for differential weighting of stimulus attributes will be necessary in order to provide some control over the "difficulty" of the decision task if this term is used to mean anything more than simply the dimensionality of the stimuli. Under Hogarth's model, task complexity is a function of both the number of attributes and disciminability between alternatives, and it is necessary to ensure that increases in the amount of information provided to decision-makers do not affect discriminability in unintended ways.

\section{A General Model of the \\ Decision-Making Process}

The failure to find between-group differences in strategy selection suggested that a more general decision-making process generated the choice times for each of the four groups. Written protocols collected from subjects were examined in an attempt to formulate some idea of the decision strategies used by subjects in this experiment. A common multistage strategy consisted of a compensatory evaluation process in conjunction with a lexicographic decision rule. In this 
strategy, several attributes were selected that had some clear compensatory relationship to one another-availability of public transportation and distance from campus, for example. Alternatives were than evaluated with respect to this subset of attributes using a compensatory combination rule. On the basis of these evaluations, some alternatives could be rejected from further consideration. In this way, the dimensionality of alternatives could be simplified into a smaller number of subsets, or factors, and utilities based on these subsets used as aspect values by which alternatives were eliminated. This decision-making strategy bears some resemblance to an elimination-by-aspects decision rule (Tversky, 1972) and may be thought of as an elimination-by-factors (EBF) choice process.

Park (1978) identified a similar process in his Operationally Simplified Satisficing-Plus model by which attribute values are simplified, or factored, to reduce cognitive complexity. In this case, for example, a 7-point scale may be recoded by the decision maker in terms of three values such as positive, neutral, or negative. In the EBF model, a similar reduction is performed in another space-the space of attributes, rather than the attribute-value space. The stimuli used in the present study were constructed so that attribute-value dimensions were already in a simplified state, often taking on values of good, fair, and poor. The extra processing spared subjects by this presentation format may have affected subsequent processing, which places some constraints on the generality of the model presented here.

An attempt was made to develop a computer model of the EBF choice process while replicating the relation observed in this study between information load and decision time. A flow chart depicting this model is shown in Figure 4.

The parameters of this model include characteristics of the decision maker, motivation and processing capacity, and parameters of the task such as the number of attributes and the number of alternatives. MAXATT is the maximum number of attributes that can be used at any one time by the decision maker in a compensatory evaluation process. This number places an upper limit on the maximum size of the attribute subsets, or the maximum amount of information that can be sampled at any one time, and is limited either by the total number of attributes in the choice task or by the processing capacity of the decision maker, whichever is smaller.

Consistent with cost-benefit models of strategy selection, motivation and processing capacity have their effect on the choice process by influencing the amount of information used in making a decision. Motiviation has an affect on the choice process by influencing the rate at which alternatives are rejected from consideration. As motivation decreases, less information is sampled before an alternative is rejected. In terms of a cost-benefit model, it might be said that when motivation is low, the marginal costs of sampling information exceed the marginal benefits of making an accurate choice.

When the number of attributes exceeds the processing capacity of the decision maker, capacity has its effect on the decision strategy not only in determining the maximum number of attributes examined at any one time but also by combining with motivation to determine the rate at which alternatives are rejected. When task complexity is high relative to the processing capacities of the decision maker, the decision task is, again, simplified by rejecting alternatives on the basis of little information.

The choice process ends when all but one alternative are eliminated or when all attributes have been exhausted, in which case a choice from among the remaining alternatives results from a final compensatory evaluation process involving all the remaining alternatives and as many attributes as the decision maker's capacity allows. It can be seen that when capacity exceeds the number of attributes and all attributes are evaluated simultaneously, the model is identical to a MAU model of decision making. On the other hand, if all attributes are evaluated in isolation, the process reduces to a lexicographic or an elimination-by-aspects choice strategy.

Simulated choice times derived from this model are shown in Figure 5. Each figure represents the average decision times of 20 hypothetical decision makers possessing the specified degrees of motivation and processing capacity. In producing these simulations, choice time was assumed to be some linear 


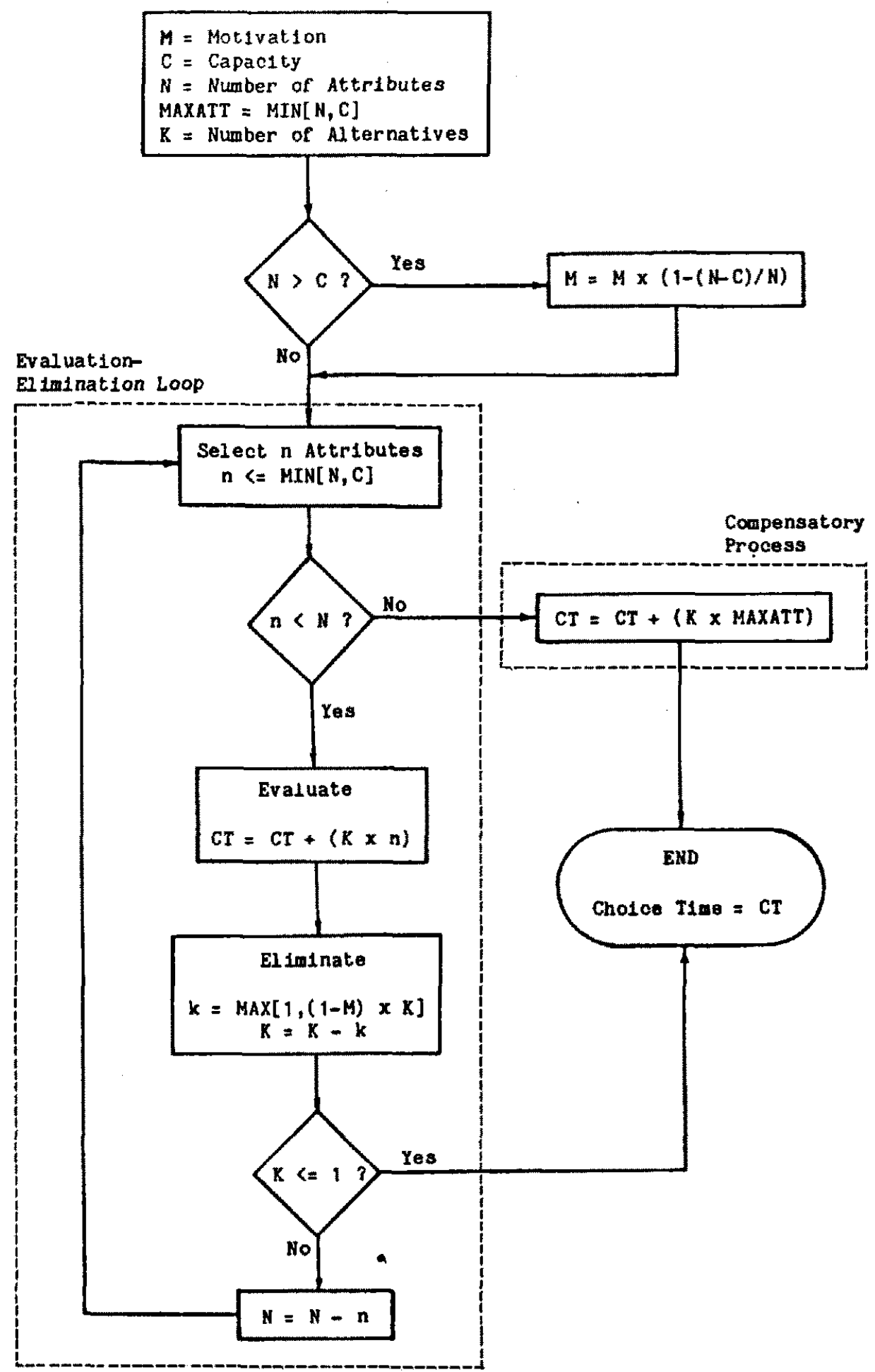

Figure 4. Multistage elimination-by-factors choice process. (MIN and MAX are functions that select the minimum and maximum values, respectively, of their arguments.) 
function of the amount of information used in making a decision. This means that any changes in choice time that are observed in response to changes in information load are a direct result of changes in the amount of information used in making a decision.

It is readily apparent that the response time functions in the simulations reproduce the five trend components most often seen in the subject analyses; response time at any level of attributes is a monotonic function of the number of alternatives; linear increases with the number of attributes are seen until the decision maker's capacity is reached; choice time is a nonmonotonic function of the number of attributes for individuals with limited processing capacities; and Linear $X$ Linear and Linear $\times$ Quadratic components are present for some individuals as well.

Whether or not the EBF process is a valid model of choice behavior in multiattribute choice tasks, it does suggest several interesting hypotheses regarding the effect of stimulus structure on strategy selection. Whether or not complex compensatory strategies are used may be a function not only of the number of attributes comprising each alternative but also of the substitutability of these attributes
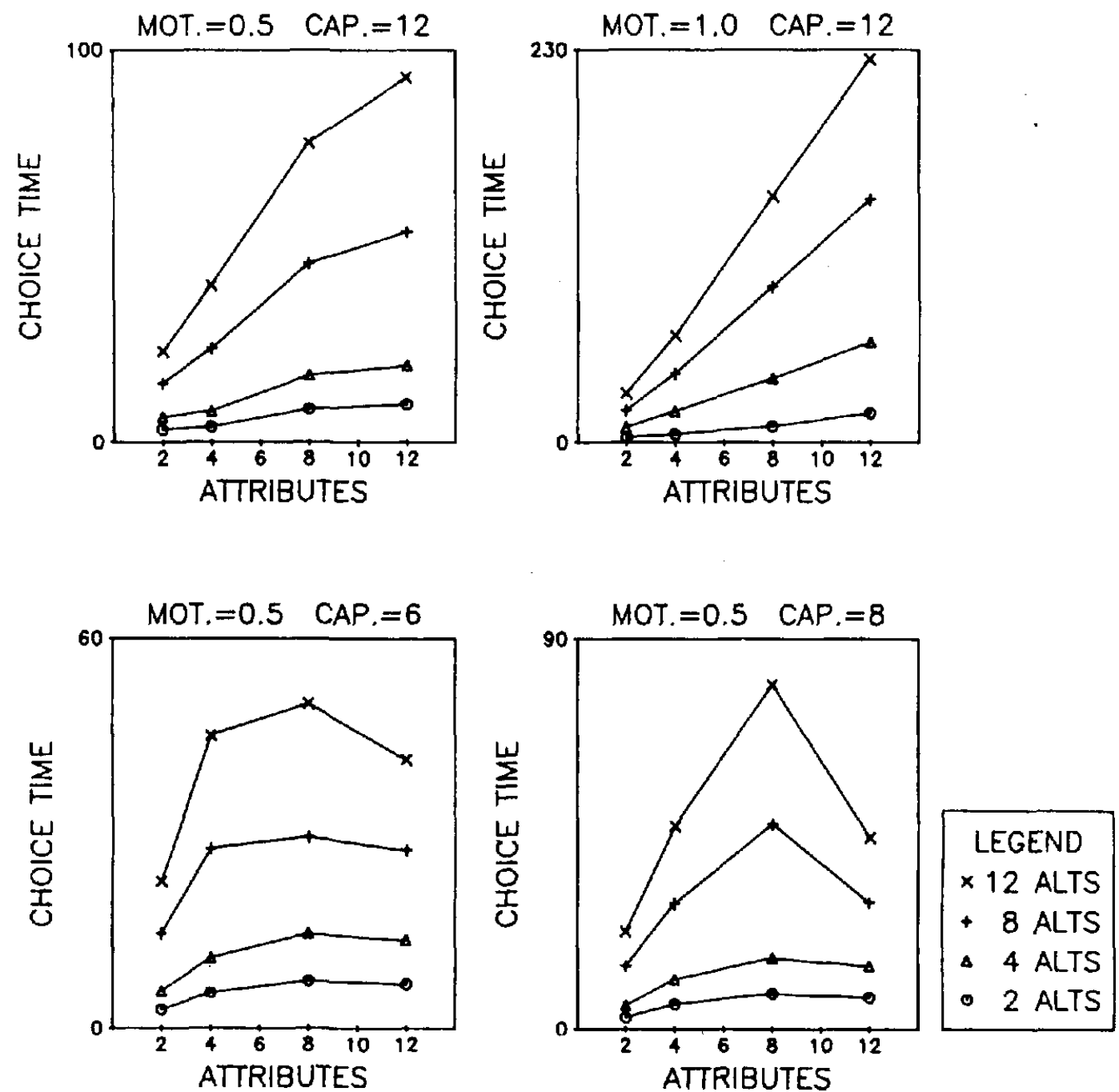

Figure 5. Choice times simulated by the elimination-by-factors model for groups of subjects possessing the indicated levels of motivation (MOT) and processing capacities (CAP). 
for one another in satisfying the needs of the decision maker. For example, when the attributes to be evaluted are the distance of an apartment from campus and the availability of transportation, a compensatory strategy may be more likely to be used than when the attributes are distance from campus and rent. In the first case, the two attributes satisfy a common need of the decision maker - to get to school easily. In the second case, not only must a decision maker trade off rent against a measure of distance, but the decision maker must also weigh his or her need for money against the need for convenience.

This suggests that the nature of task complexity involves more than simply the number of attributes. The needs of the decision maker and the extent to which attributes are substitutable in satisfying these needs are also likely to be important determinants of task complexity and the degree to which cognitive simplification strategies are used.

The use of choice time as a dependent variable in this experiment demonstrates the necessity of convergent operations in the investigation of decision-making processes. Should the results reported here prove replicable, results from process-tracing operations such as information search (Payne, 1976) and protocol analysis (Payne, Braunstein, \& Carroll, 1978) will have to be reconciled with measures of response time. For example, previous findings that the number of alternatives has a greater impact on the tendency to use simplification strategies than does the number of attributes (Payne, 1982) were unsupported in this study.

If decision time is to be used as an alternative measure of strategy complexity, more information regarding the effects of task complexity, information load, and strategy on choice time is needed. An assumption was made in the EBF choice model that decision time was a linear function of the amount of information used in making a decision. The validity of this assumption is not known. One possible way of studying this would be to train subjects to use various decision strategies and to examine changes in decision time that occur with increases in information load. If there is some way in which the adequacy of decisions can also be evaluated, this information, in conjunction with decision times, could be used to assess the efficiency of various strategies for processing information.

In recent information-processing approaches to the study of intelligence, the decomposition of intellectual tasks into discrete stages, such as "componential analysis" (Sternberg, 1977), has proved useful in increasing the validity of response time as a measure of cognitive processing as well as in revealing individual differences in ability at the level of elementary operations. Similar approaches are conceivably applicable to decision-making tasks. Movement has been made in this direction through attempts to decompose decision strategies into elementary operations (Huber, 1980), through the development of computer models of common decision strategies (Klayman, 1982), and through the use of response time to investigate the organization of information in memory (Johnson \& Russo, 1978).

\section{References}

Christensen-Szalanski, J. J. J. (1978). Problem solving strategies: A selection mechanism, some implications and some data. Organizational Behavior and Human Performance, 22, 307-323.

Christensen-Szalanski, J. J. J. (1980). A further examination of the selection of problem-solving strategies: The effects of deadlines and analytic aptitudes. Organizational Behavior and Human Performance, 25, $107-$ 122.

Einhorn, H. J. (1970). The use of nonlinear, noncompensatory models in decision making. Psychological Bulletin. 73, 221-230.

Einhorn, H. J. (1971). The use of nonlinear, noncompensatory models as a function of task and amount of information. Organizational Behavior and Human Performance, 6, 1-27.

Eysenck, H. J., \& Eysenck, S. B. G. (1964). Eysenck personality inventory. San Diego, CA: Educational and Industrial Testing Service.

Grether, D. M., \& Plott, C. R. (1979), Economic theory of choice and the preference reversal phenomenon. American Economic Review, 69, 623-638.

Hendrick, C., Mills, J., \& Kiesler, C. A. (1968). Decision time as a function of the number and complexity of equally attractive alternatives. Journal of Personality and Social Psychology, 8, 313-318.

Hogarth, R. M. (1975). Decision time as a function of task complexity. In Wendt \& Vlek (Eds.), Utility, probability and human decision making (pp. 321338). Dordrecht-Holland: D. Reidel.

Huber, O. (1980). The influence of some task variables on cognitive operations in an information-processing decision model. Acta Psychologica, 45, 187-196.

Jacoby, J., Speller, D. E., \& Berning, C. K. (1975). Brand 
choice behavior as a function of information load: Replication and extension. Journal of Consumer Research, 1, 33-42.

Jacoby, J., Speller, D. E., \& Kohn, C. A. (1974). Brand choice behavior as a function of information load. Journal of Marketing Research, 11, 62-69.

Johnson, E. J., \& Russo, J. E. (1978). The organization of product information in memory identified by recall times. In H. Keith Hunt (Ed.), Advances in consumer research (pp. 79-86). Ann Arbor, MI: Ann Arbor Association for Consumer Research.

Kiesler, C. A. (1966). Conflict and number of choice alternatives. Psychology Reports, 18, 603-610.

Klayman, J. (1982). Simulation of six decision strategies: Comparisons of search patterns, processsing characteristics, and response to task complexity. Unpublished manuscript, University of Chicago, Graduate School of Business, Center for Decision Research.

Park, C. W. (1978). A seven-point scale and a decisionmaker's simplifying choice strategy. An operationalized satisficing-plus model. Organizational Behavior and Human Performance, 21, 252-271.

Payne, J. W. (1976). Task complexity and contingent processing in decision making: An information search and protocol analysis. Organizational Behavior and Human Performance, 16, 366-387.

Payne, J. W. (1982). Contingent decision behavior. Psy. chological Bultetin, 92, 382-402.

Payne, J. W., \& Braunstein, M. L. (1978). Risky choice: An examination of information acquisition behavior. Memory \& Cognition, 5, 554-561.

Payne, J. W., Braunstein, M. L., \& Carroll, J. S. (1978). Exploring pre-decisional behavior: An alternative apm proach to decision research. Organizational Behavior and Human Performance, 22, 17-44.
Pollay, R. W. (1970). A model of decision times in difficult decision situations. Psychological Review, 77, 274-281.

Russo, J. E., \& Dosher, B. A. (1983). Strategies for multiattribute binary choice. Journal of Experimental Psychology: Learning, Memory, and Cognition, 9, 676696.

Shugan, S. M. (1980a). The cost of thinking. Journat of Consumer Research 7, 99-111.

Shugan, S. M. (1980b, February). The cost of thinking: Its implications. Paper presented at the Interdisciplinary Choice Theory Conference, University of Washington.

Simon, H. A. (1955). A behavioral model of rational choice. Quarterly Journal of Economics, 69, 99-118.

Simon, H. A. (1979). Rational decision making in business organizations. American Economic Review; 69, 493513.

Sternberg, R. J. (1977). Intelligence, information processing, and analogical reasoning. The componential analysis of human abilities. Hillsdale, NJ: Erlbaum.

Svenson, O. (1979), Process descriptions of decison making. Organizational Behavior and Human Peformance, 16, 366-387.

Tversky, A. (1972). Elimination by aspects: A theory of choice. Psychological Review. 79, 281-299.

Weitzman $_{x}$ M. L. (1979), Optimal search for the best alternative. Econometrica, 47, 641-654.

Wright, P. (1975). Consumer choice strategies: Simplifying vs. optimizing. Journal of Marketing Research, 11,60 67.

Received October 15,1983 Revision received August 2, 1984

\section{Correction to Kelso et al.}

In the article "Functionally Specific Articulatory Cooperation Following Jaw Perturbations During Speech: Evidence for Coordinative Structure" by J. A. Scott Kelso, Betty Tuller, E. VatikiotisBateson, and Carol A. Fowler (Journal of Experimental Psychology: Human Perception and Performance, 1984, Vol. 10, No. 6, pp. 812832 ), there is a typographical error on page 818 (line 3 , right-hand column): "( $S D=18 \mathrm{~ms})$ " should read " $(S D=7 \mathrm{~ms})$." 